\title{
Stereotactic body radiation therapy for the management of HCC
}

\author{
Jia-Xin Li, Hong Wu, Yong Zeng \\ Department of Liver Surgery, West China Hospital of Sichuan University, Chengdu 610041, Sichuan, China.
}

Correspondence to: Prof. Yong Zeng, Department of Liver Surgery, West China Hospital of Sichuan University, Chengdu 610041, Sichuan, China. E-mail: zengyong@medmail.com.cn

How to cite this article: Li JX, Wu H, Zeng Y. Stereotactic body radiation therapy for the management of HCC. Hepatoma Res 2020;6:39. http://dx.doi.org/10.20517/2394-5079.2020.13

Received: 10 Feb 2020 First Decision: 27 Mar 2020 Revised: 28 May 2020 Accepted: 8 Jun 2020 Published: 10 Jul 2020

Academic Editor: Ying-Hong Shi Copy Editor: Cai-Hong Wang Production Editor: Tian Zhang

\begin{abstract}
Hepatocellular carcinoma (HCC) is a common malignant tumor in China. After years of efforts, there has been great progress in the management of liver cancer, but overall, it is still not ideal. At present, there are many therapies for liver cancer, including surgical resection, transcatheter arterial chemoembolization (TACE), ablation, molecular targeted therapy, stereotactic body radiation therapy, chemotherapy, immunotherapy, and so on. Studies have reported that TACE combined with radiotherapy can shrink the tumor, and some of the remainder will be resectable, resulting in cure. For HCC with tumor thrombus, the tumor thrombus was reduced and then resected after neoadjuvant radiotherapy. The survival time of the patients with portal vein tumor thrombus was significantly longer than that of the patients without neoadjuvant radiotherapy. Large liver cancer will be reduced to small liver cancer after comprehensive treatment, which can be transformed into stereotactic radiotherapy or radiofrequency ablation, and can also be palliative to radical treatment. Individualized and multidisciplinary therapy for liver cancer is the direction of future development. More clinical evidence-based level of radiotherapy treatment of liver cancer should be done in the future.
\end{abstract}

Keywords: Hepatocellular carcinoma, stereotactic body radiation therapy, tumor thrombus, combined therapy

\section{INTRODUCTION}

Hepatocellular carcinoma (HCC) is a common malignant tumor that seriously endangers human health. At present, the main treatment for small HCC is surgical resection and radiofrequency ablation, but some patients are not suitable for surgery or radiofrequency ablation. In recent years, with the development of radiotherapy technology, the application of stereotactic body radiation therapy (SBRT) is increasing. Some retrospective studies have shown that its therapeutic effect on small liver tumors is equivalent to that of traditional surgery or radiofrequency ablation, and that it can also be used as a radical treatment

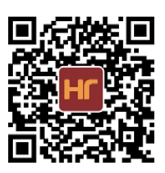


for small liver tumors. Some patients with unresectable tumor thrombus can be converted to surgical resection after radiotherapy, and external radiotherapy can also be applied for bridging treatment before liver transplantation. A narrow margin with a distance of less than $1 \mathrm{~cm}$ from tumor resection edge is possible with radiotherapy after liver resection, to reduce local recurrence or distant metastasis of HCC and to prolong disease-free survival (DFS) of patients. The mechanism of killing tumor cell by SBRT may be different from conventional fractionated radiotherapy. With the increase of fractionated dose, double-strand DNA breaks increase, and the repair of sublethal damage decreases. Moreover, when the single fractionated dose is more than 8-10 Gy, the tumor vascular endothelial cells rapidly wither after 1-6 h of irradiation, and blood vessels are occluded, leading to a secondary tumor killing effect, which is mainly through the acid sphingomyelinase pathway-mediated apoptosis of vascular endothelial cells ${ }^{[1-3]}$, but conventional radiotherapy does not have the above effect. After conventional fractionated radiotherapy, tumor cell death is mainly achieved by cell apoptosis, while after high dose irradiation, tumor cell death is mainly in the form of "swelling death" ${ }^{[4]}$, and tumor cell membrane is destroyed, releasing tumor specific antigen, becoming "in situ tumor vaccine", which can stimulate the immune system to kill residual tumor ${ }^{[5]}$.

\section{EFFECT OF SBRT ON LIVER FUNCTION}

Cárdenes et al. ${ }^{[6]}$ conducted a phase I clinical study of dose escalation in patients with liver cancer: patients with a tumor diameter less than $6 \mathrm{~cm}$ and Child-Pugh (CP) Class A could tolerate 48 Gy in three fractions, and the patients with CP class B could not tolerate 42 Gy in three fractions, while no adverse effects occurred after adjustment to $40 \mathrm{~Gy}$ in 5 fractions. The $\mathrm{CP}$ score was the only risk factor related to hepatotoxicity or death within six months of SBRT treatment $(P=0.03)$. Three patients had classic radiation-induced liver disease, all of which were above CP class B7. We believe that good liver function was needed for SBRT treatment of liver cancer. Furthermore, $\mathrm{CP}$ score must be $\leq 7$, and patients with $\mathrm{CP}$ score $>8$ have high risk of liver failure after SBRT treatment.

\section{SBRT FOR PRIMARY LIVER CANCER}

Many studies in the literature have focused on the study of liver cancer lesions with the diameter of less than $6 \mathrm{~cm}$. The 1- and 2-year local control (LC) rates after SBRT were $72 \%-100 \%$ and $54.4 \%-100 \%$, respectively, and the 1 -year survival rates were $72.7 \%-100 \%$ and $45.3 \%-87.9 \%$, respectively ${ }^{[6-17]}$, but the dose fractionation of radiotherapy used in various clinical studies was not the same. Patients with CP class A or tumor diameter less than $3 \mathrm{~cm}$ usually undergo irradiation more than 10 Gy each time, total for 3-5 times. Because of the good LC rates and tolerability, SBRT is recognized as an alternative for small liver tumors unsuitable for surgery. Yoon et al. ${ }^{[7]}$ applied SBRT to treat 93 cases of inoperable liver cancer with median diameter of $2 \mathrm{~cm}$ (1-6 cm), given 30-60 Gy or 10-20 Gy in 3-4 fractions, and the 1- and 3-year overall survival (OS) rates were 86 and $53.8 \%$, respectively. LC failure was mainly seen in patients with liver cancer $\geq 3 \mathrm{~cm}$, and the 3 -year LC rates of liver cancer with diameter $\geq 3.0,2.1-3.0$ and $\leq 2.0 \mathrm{~cm}$ were $76.3,93.3$ and $100 \%$, respectively, the intrahepatic 3 -year recurrence-free survival (RFS) rate was $32.4 \%$. Six patients (6.5\%) had $\geq$ grade 3 hepatotoxicity. Similarly, Kwon et al. ${ }^{[8]}$ reported that 42 cases of liver cancer were treated with CyberKnife at a dose of 30-39 Gy in 3 fractions; the complete response (CR) rate was 59.6\% and the partial response (PR) rate was $26.2 \%$, while the 1 - and 3-year OS rates were 92.9 and $58.6 \%$, respectively. Kimura et al. ${ }^{[9]}$ used $48 \mathrm{~Gy}$ in 4 fractions to treat 65 patients with CP class A/B and median tumor diameter of $1.6 \mathrm{~cm}$; 2-year OS, RFS and LC were 76,40 and $100 \%$. Fifteen patients (23\%) had $\geq$ grade 3 adverse reactions $6-12$ months after $\mathrm{SBRT}$, and the level $\geq 3$ adverse reactions in $\mathrm{CP}$ class $\mathrm{B}$ were higher than those in $\mathrm{CP}$ class $\mathrm{A}(P=0.0127)^{[9]}$.

There is no prospective study comparing SBRT with surgery or TACE in the treatment of primary liver cancer. Honda et al. ${ }^{[15]}$ applied sequential SBRT and TACE compared with TACE alone in the treatment of primary liver cancer with diameter less than $3 \mathrm{~cm}$; of the 30 cases in the SBRT group, 29 cases (96.3\%) had a CR, but only 1 in 38 cases in the TACE group had a CR (3.3\%). There was no radiation-related injury in 
Table 1. Studies of stereotactic body radiation therapy in hepatocellular carcinoma

\begin{tabular}{|c|c|c|c|c|c|c|c|}
\hline Authors & Cases & $\begin{array}{c}\text { CP class A/ } \\
\text { B/C }\end{array}$ & $\begin{array}{l}\text { Tumor diameter } \\
\text { or GTV }\end{array}$ & $\begin{array}{c}\text { Dose/times (Gy/ } \\
\text { time) }\end{array}$ & $\begin{array}{c}\text { CR/PR/SD } \\
\text { (\%) }\end{array}$ & $\begin{array}{c}\text { 1/2/3-year LC } \\
\text { (\%) }\end{array}$ & $\begin{array}{l}\text { 1/2/3-year } \\
\text { OS (\%) }\end{array}$ \\
\hline Cárdenes et al. $^{[6]}$ & 17 & A6/B11 & $2-6 \mathrm{~cm}$ or $8-95 \mathrm{~cm}^{3}$ & $36-48 / 3 ; 36-42 / 3 ; 40 / 5$ & $25 / 56 / 19$ & $100 / \mathrm{N} / \mathrm{N}$ & $75 / 60 / \mathrm{N}$ \\
\hline Yoon et al. ${ }^{[7]}$ & 93 & A69/B24 & $1-6 \mathrm{~cm}$ & $30-60 / 3-4$ & $15.5 / 45.7 / 36.9$ & $94.8 / N / 92.1$ & $86 / N / 53.8$ \\
\hline Kimura et al. ${ }^{[9]}$ & 65 & A56/B9 & $0.5-5.4 \mathrm{~cm}^{3}$ & $48 / 4$ or $60 / 8$ & $100 / 100 / \mathrm{N}$ & $92.3 / 76 / \mathrm{N}$ & $\mathrm{N}$ \\
\hline Andolino et al. ${ }^{[12]}$ & 60 & A36/B24 & $1-6.5 \mathrm{~cm}$ & $\mathrm{~m} 44 / \mathrm{m} 3 ; \mathrm{m} 40 / \mathrm{m} 5$ & $30 / 40 / 25$ & $\mathrm{~N} / 90 / \mathrm{N}$ & $N / 67 / N$ \\
\hline Takeda et al. ${ }^{[13]}$ & 63 & A44/B19 & $1-5 \mathrm{~cm}$ & $35-40 / 5$ & $100 / 95 / 92$ & $100 / 87 / 73$ & $\mathrm{~N}$ \\
\hline Park et al. ${ }^{[16]}$ & 26 & A19/B7 & $1.1-5.7 \mathrm{~cm}$ & $40-50 / 10$ & $25 / 42.9 / 32.1$ & $\mathrm{~N} / 87.6 / \mathrm{N}$ & $88.5 / 67.2 / \mathrm{N}$ \\
\hline Yuan et al. ${ }^{[17]}$ & 22 & $\mathrm{~A} 10 / \mathrm{B} 10 / \mathrm{C} 2$ & $1.6-9.5 \mathrm{~cm}$ & $39-54 / 3-8$ & $50 / 41 / 9$ & $92.9 / 90 / 67.7$ & $\mathrm{~N}$ \\
\hline
\end{tabular}

CP: Child-Pugh Class; GTV: gross tumor volume; m: median; N: not applicable; CR: complete response; PR: partial response; SD: stable disease; LC: local control; OS: overall survival

the SBRT group. Another retrospective study compared CyberKnife with surgical resection in the treatment of stage I liver cancer; 22 cases were treated with SBRT, and 26 patients achieved Ro resection. There was no difference in 3-year survival rate $(69.2 \%$ vs. $57.1 \%, P=0.49)$. Although the sample size was limited, the authors believed that SBRT is effective in the treatment of early stage liver cancer and that the curative effect is not inferior to that of surgical resection ${ }^{[17]}$ [Table 1].

\section{SBRT IN THE TREATMENT OF PORTAL/VENA CAVA TUMOR THROMBUS}

Tumor thrombus is an unavoidable difficulty in the treatment of liver cancer. The tumor thrombus will aggravate the occurrence of portal hypertension, ascites, liver failure, intrahepatic and extrahepatic dissemination. Choi et al. ${ }^{[18]}$ analyzed the treatment of advanced liver cancer with portal vein tumor thrombus (PVTT) by SBRT combined with TACE; 9 cases with PVTT treated with TACE at a median dose of 36 Gy (30-39 Gy in 3 fractions), and after a median follow-up time of 10.5 months, achieved PVTT CR rate of $11.1 \%(1 / 9)$, PR rate of $33.3 \%$ (3/9), objective response rate (ORR) of $44.4 \%$ (4/9) and median OS of 8 months in advanced liver cancer ${ }^{[18]}$. Xi et al ${ }^{[19]}$ applied a median dose of $36 \mathrm{~Gy}$ in 6 fractions to treat primary liver cancer with portal/vena cava tumor thrombus, and 25 of 41 patients had received TACE, with a median follow-up of 10 months. The CR, PR, stable disease (SD), progressive disease (PD) of tumor thrombus were 36.6, 39, 17 and 7\%, respectively. The 1-year OS was 50.3\%, and the median OS was 13 months. Only one patient had an increased bilirubin level.

Wu et al. ${ }^{[20]}$ reported TACE combined with SBRT with 4-8 Gy per fraction for advanced liver cancer with portal/vena cava tumor thrombus. After 4-6 weeks, CR was 8.6\%, PR 42.8\%, SD 48.6\%, and 1-, 2-, 3-year OS were 59.3, 31.6 and $26.6 \%$, respectively, while median survival time was 11 months. Tse et al. ${ }^{[21]}$ recruited 16 cases of primary liver cancer with PVTT, applied an average dose of 36 Gy in 6 fractions, achieved CR of $6 \%$, PR of $19 \%$ and SD of $38 \%$. Bujold et al. ${ }^{[22]}$ reported on 56 patients with PVTT who were treated with an average dose of $36 \mathrm{~Gy}$ (24-54 Gy in 6 fractions), where the 1- and 2-year OS rates were 44 and 27\%, respectively. Multivariate analysis showed that venous tumor thrombus was the worst prognostic factor, $\mathrm{HR}=2.47(P=0.01)$.

\section{PREOPERATIVE NEOADJUVANT SBRT FOR HCC}

Patients with HCC (China liver cancer staging, CNLC IIIa) undergo surgical resection, but only a small part of the patients have long-term survival, and most of the patients have recurrence or metastasis in the short term, leading to death. Radiotherapy alone for PVTT is a palliative therapy. Kamiyama et al. ${ }^{[23]}$ reported on patients with HCC complicated with tumor thrombus who received preoperative neoadjuvant radiotherapy, which was more effective than simple operation. The first branch or main tumor thrombus of portal vein was treated with radiotherapy at 30-36 Gy/10-12 times. After radiotherapy, the thrombus and intrahepatic lesions were removed simultaneously within 2 weeks. Intervention, radiofrequency, anhydrous 
alcohol injection and other treatments were given during the follow-up periods. The results showed that the median survival time was 19.6 months for the patients who received surgery combined with external radiotherapy, and 9.1 months for the patients who did not undergo surgery. There was a significant difference between the two groups $(P=0.036)$. The pathology of the surgical specimens showed that $83 \%$ of the tumor thrombi were completely necrotic (completely relieved of disease). Therefore, the main purpose of surgery is to improve the control rate of primary HCC and dredge the portal vein. For patients with tumor thrombus, preoperative radiotherapy combined with surgical removal of thrombus is an effective method of comprehensive treatment. A randomized, prospective, multicenter clinical study was conducted in Shanghai Oriental Hepatobiliary Surgery Hospital to compare the survival of patients with HCC and PVTT before operation with or without neoadjuvant radiotherapy ${ }^{[24]}$. A total of 82 patients in the radiotherapy group received $18 \mathrm{~Gy} / 6$ times of neoadjuvant external radiotherapy; 82 patients in the control group did not receive neoadjuvant radiotherapy. The results showed that the OS rates of radiotherapy group and operation group were $89.0 \% v$ s. $81.7 \%, 75.2 \% v s .43 .1 \%, 43.9 \% v s .16 .7 \%$ and $27.4 \% v s .9 .4 \%$ at $6,12,18$ and 24 months, respectively $(P<0.001)$. The DFS rates of the two groups were $56.9 \%$ vs. $42.1 \%, 33.0 \% v s .14 .9 \%, 20.3 \% v s$. $5.0 \%$ and $13.3 \%$ vs. $3.3 \%(P<0.001)^{[24]}$. Neoadjuvant radiotherapy can significantly improve the postoperative survival of patients with HCC.

\section{TRANSFORMATION SBRT FOR HCC}

Kim et al. ${ }^{[25]}$ of Yonsei University in South Korea reviewed the effect of concurrent radiotherapy and chemotherapy on patients with HCC. A total of 264 patients were selected, most of whom received threedimensional conformal radiotherapy because of PVTT or residual liver volume insufficiency. Most of them received radiotherapy with a single dose of $1.8 \mathrm{~Gy}$ and a total dose of $45 \mathrm{~Gy}$, and 5-fluorouracil (5-FU) was infused in the first and fifth week simultaneous with radiotherapy. One month after radiotherapy, 5-FU and cisplatin were infused once every 4 weeks for 3-12 cycles. Among them, 18 cases were converted into resectable cases. Postoperative pathology showed that 4 cases $(22.2 \%)$ were completely necrotic and 7 cases (38.9\%) were 70\%-99\% necrotic. Among the 18 patients who underwent surgery, the median survival time and median progression time were 40 and 24 months, respectively, and the median DFS time of the 4 patients with complete tumor necrosis was 54.6 months. Therefore, after conformal radiotherapy, some patients with unresectable HCC could be converted to resectable HCC.

\section{BRIDGING SBRT BEFORE LIVER TRANSPLANTATION}

Orthotopic liver transplantation is the most effective treatment for HCC patients with liver transplantation indications. However, due to the limited number of liver donors, many patients may have tumor progression while on the long waiting list, thus losing the best opportunity for liver transplantation. Therefore, bridging therapy to delay tumor progression is very important. SBRT can be used as a bridge treatment for liver cancer patients waiting for liver transplantation. It is neither a new adjuvant radiotherapy nor a transformation radiotherapy. Because SBRT belongs to radical treatment, the majority of HCC patients with $\mathrm{T} 1$ or $\mathrm{T} 2$ can be reduced to To. The purpose of liver transplantation is to replace the decompensated liver with normal liver function. The purpose of radiotherapy is to control tumor progression while lacking a liver donor.

Eighteen patients with liver cancer who received SBRT before transplantation were reported by Rochester University Medical Center and William Beaumont Hospital in Michigan ${ }^{[26,27]}$. The median dose of radiotherapy was $50 \mathrm{~Gy} / 10$ times. There was no serious gastrointestinal adverse reactions or radiation hepatitis. The median waiting period after radiotherapy was 6.3 months. Twelve patients received liver transplantation successfully, and 10 patients showed complete pathological necrosis. The median followup period was 19.6 months, and all patients survived. Therefore, SBRT is a safe and effective treatment for liver cancer patients waiting for liver transplantation. It can control the tumor and relieve the pressure of a liver donor before liver transplantation. A study reported ${ }^{[28]}$ on 379 patients treated with pre-transplantation 
bridging therapy, including 36 patients with SBRT, 99 patients with TACE and 244 patients with radiofrequency ablation (RFA). Finally, 312 patients received liver transplantation, 30 in the SBRT group, 79 in the TACE group, and 203 in the RFA group. The 1-, 3- and 5-year survival rates were $83 \%, 61 \%$ and $61 \%$ in the SBRT group, 86,61 and $56 \%$ in the TACE group, and $86 \%, 72 \%$ and $61 \%$ in the RFA group, respectively $(P=0.4)$. SBRT is as safe and effective as TACE and RFA in the treatment of HCC before transplantation. Compared with TACE and RFA, SBRT has more advantages in the treatment of HCC patients with ascites and prolonged prothrombin time.

\section{POSTOPERATIVE ADJUVANT SBRT FOR HCC}

Liver cancer with the distance of less than $1 \mathrm{~cm}$ from the bifurcation of the portal vein, the confluence of three main hepatic veins and inferior vena cava, is at high risk of recurrence after liver resection. The 5-year recurrence rate is reported to be more than $90 \%$. At present, there is no effective adjuvant therapy to reduce such a high recurrence rate of this kind of liver cancer. Wang et al. ${ }^{[29]}$ reported the results of radiotherapy for the first time in patients with narrow margin surgery of central type liver cancer, who had retained a silver positioning mark in the operation area guided by postoperative adjuvant radiotherapy.

From 2007 to 2010, 181 patients were analyzed retrospectively. They were divided into three groups: group A: narrow margin surgery combined with postoperative radiotherapy (33 patients), group B: narrow margin surgery ( 83 patients) and group C: wide margin surgery with cutting edge more than $1 \mathrm{~cm}$ (65 patients), and postoperative radiotherapy dose was 46-60 Gy/23-30 times, with a median dose of $56 \mathrm{~Gy}$; the 3-year OS of groups A, B and C was 89.1, 67.7 and 86.0\%, respectively, while DFS was 64.2, 52.2 and $60.1 \%$, respectively. Group A had similar OS $(P=0.957)$ and DFS $(P=0.972)$ as group C. Compared with group $\mathrm{B}$, the OS $(P=0.009)$ and DFS $(P=0.038)$ of group A showed significant advantages The incidence of grade-3 adverse reactions in group A was $12.1 \%$ and no more than grade- 4 adverse reactions occurred. The study showed that postoperative radiotherapy can make up for the deficiency of narrow margin surgery, and that the effect of narrow margin surgery combined with postoperative radiotherapy can achieve asimilar effect as wide margin radical surgery without serious adverse reactions.

Microvascular invasion (MVI) is the most important risk factor for early postoperative recurrence of HCC, which has been proved to be an independent predictor of OS and $\mathrm{DFS}^{[30]}$. Even for patients with small liver cancer, MVI can increase tumor recurrence rate and significantly reduce OS, and MVI can only be detected by postoperative histological examination.

Wang et al. ${ }^{[31]}$ reviewed and compared the results of conservative therapy (CT), TACE and radiotherapy in 136 HCC patients with MVI. Narrow margin rate in the radiotherapy group was significantly higher than in the other two groups $(P=0.010)$. Postoperative radiotherapy dose was 54-60 Gy/23-30 times, where the results showed that there were significant differences between the radiotherapy group and TACE group and the radiotherapy group and CT group in DFS $(P<0.05)$, and the radiotherapy group and TACE group and the RT group and CT group in OS $(P<0.05)$. Subgroup analysis based on MVI degree and surgical margin showed that RFS and OS of patients in the radiotherapy group with narrow surgical margin were significantly longer than those of the TACE and CT groups, but not related to MVI degree. There was no significant difference in survival outcome between the three groups with wide surgical margin.

\section{SUMMARY}

To sum up, surgery is an effective way to cure HCC in the early and middle stages. The high recurrence rate and the lack of effective rescue treatment make the curative effect not very ideal. Although the related research results of surgery combined with SBRT are limited, they all show that it is a safe and effective treatment. As a new way of radiotherapy, preoperative SBRT has shown its unique advantages in the adjuvant 
treatment of liver cancer. In a word, it is safe and feasible to combine preoperative and postoperative radiotherapy in the era of multidisciplinary comprehensive treatment, and it has also improved the curative effect. However, more in-depth research is needed to further determine whether it can make HCC patients benefit more from it.

\section{DECLARATIONS}

\section{Authors' contributions}

Data analysis and interpretation and paper writing: Li JX

Conception and design of the study: Wu $\mathrm{H}$, Zeng Y

\section{Availability of data and materials}

Not applicable.

\section{Financial support and sponsorship}

None.

\section{Conflicts of interest}

All authors declared that there are no conflicts of interest.

\section{Ethical approval and consent to participate}

Not applicable.

\section{Consent for publication}

Not applicable.

\section{Copyright}

(c) The Author(s) 2020.

\section{REFERENCES}

1. Garcia-Barros M, Paris F, Cordon-Cardo C, Lyden D, Rafii S, et al. Tumor response to radiotherapy regulated by endothelial cell apoptosis. Science 2003;300:1155-9.

2. Kolesnick R, Fuks Z. Radiation and ceramide-induced apoptosis. Oncogene 2003;22:5897-906.

3. Park HJ, Griffin RJ, Hui S, Levitt SH, Song CW. Radiation-induced vascular damage in tumors: implications of vascular damage in ablative hypofractionated radiotherapy (SBRT and SRS). Radiat Res 2012;177:311-27.

4. Würthwein G, Klingebiel T, Krümpelmann S, Metz M, Schwenker K, et al. Population pharmacokinetics of high-dose etoposide in children receiving different conditioning regimens. Anticancer Drugs 2002;13:101-10.

5. Lee Y, Auh SL, Wang Y, Burnette B, Wang Y, et al. Therapeutic effects of ablative radiation on local tumor require CD8+ T cells: changing strategies for cancer treatment. Blood 2009;114:589-95.

6. Cárdenes HR, Price TR, Perkins SM, Maluccio M, Kwo P, et al. Phase I feasibility trial of stereotactic body radiation therapy for primary hepatocellular carcinoma. Clin Transl Oncol 2010;12:218-25.

7. Yoon SM, Lim YS, Park MJ, Kim SY, Cho B, et al. Stereotactic body radiation therapy as an alternative treatment for small hepatocellular carcinoma. PLoS One 2013;8:e79854.

8. Kwon JH, Bae SH, Kim JY, Choi BO, Jang HS, et al. Long-term effect of stereotactic body radiation therapy for primary hepatocellular carcinoma ineligible for local ablation therapy or surgical resection. Stereotactic radiotherapy for liver cancer. BMC Cancer 2010;10:475.

9. Kimura T, Aikata H, Takahashi S, Takahashi I, Nishibuchi I, et al. Stereotactic body radiotherapy for patients with small hepatocellular carcinoma ineligible for resection or ablation therapies. Hepatol Res 2015;45:378-86.

10. Scorsetti M, Comito T, Cozzi L, Clerici E, Tozzi A, et al. The challenge of inoperable hepatocellular carcinoma (HCC): results of a singleinstitutional experience on stereotactic body radiation therapy (SBRT). J Cancer Res Clin Oncol 2015;141:1301-9.

11. Sanuki N, Takeda A, Oku Y, Mizuno T, Aoki Y, et al. Stereotactic body radiotherapy for small hepatocellular carcinoma: a retrospective outcome analysis in 185 patients. Acta Oncol 2014;53:399-404.

12. Andolino DL, Johnson CS, Maluccio M, Kwo P, Tector AJ, et al. Stereotactic body radiotherapy for primary hepatocellular carcinoma. Int J Radiat Oncol Biol Phys 2011;81:e447-53.

13. Takeda A, Sanuki N, Eriguchi T, Kobayashi T, Iwabutchi S, et al. Stereotactic ablative body radiotherapy for previously untreated solitary 
hepatocellular carcinoma. J Gastroenterol Hepatol 2014;29:372-9.

14. Bae SH, Park HC, Lim DH, Lee JA, Gwak GY, et al. Salvage treatment with hypofractionated radiotherapy in patients with recurrent small hepatocellular carcinoma. Int J Radiat Oncol Biol Phys 2012;82:e603-7.

15. Honda Y, Kimura T, Aikata H, Kobayashi T, Fukuhara T, et al. Stereotactic body radiation therapy combined with transcatheter arterial chemoembolization for small hepatocellular carcinoma. J Gastroenterol Hepatol 2013;28:530-6.

16. Park JH, Yoon SM, Lim YS, Kim SY, Shim JH, et al. Two-week schedule of hypofractionated radiotherapy as a local salvage treatment for small hepatocellular carcinoma. J Gastroenterol Hepatol 2013;28:1638-42.

17. Yuan Z, Tian L, Wang P, Song Y, Dong Y, et al. Comparative research on the efficacy of CyberKnife ${ }^{\circledR}$ and surgical excision for Stage I hepatocellular carcinoma. Onco Targets Ther 2013;6:1527-32.

18. Choi BO, Choi IB, Jang HS, Kang YN, Jang JS, et al. Stereotactic body radiation therapy with or without transarterial chemoembolization for patients with primary hepatocellular carcinoma: preliminary analysis. BMC Cancer 2008;8:351.

19. Xi M, Zhang L, Zhao L, Li QQ, Guo SP, et al. Effectiveness of stereotactic body radiotherapy for hepatocellular carcinoma with portal vein and/or inferior vena cava tumor thrombosis. PLoS One 2013;8:e63864.

20. Wu DH,Chen LH. Efficacy of 3 dimensional conformal hypofractionated single high dose radiotherapy combined with transcatheter arterial chemoembolization for portal vein tumor thrombus in patients with hepatocellular carcinoma. Chin J Cancer 2004;23:3.825s.

21. Tse RV, Hawkins M, Lockwood G, Kim JJ, Cummings B, et al. Phase I study of individualized stereotactic body radiotherapy for hepatocellular carcinoma and intrahepatic cholangiocarcinoma. J Clin Oncol 2008;26:657-64.

22. Bujold A, Massey CA, Kim JJ, Brierley J, Cho C, et al. Sequential phase I and II trials of stereotactic body radiotherapy for locally advanced hepatocellular carcinoma. J Clin Oncol 2013;31:1631-9.

23. Kamiyama T, Nakanishi K, Yokoo H, Tahara M, Nakagawa T, et al. Efficacy of preoperative radiotherapy to portal vein tumor thrombus in the main trunk or first branch in patients with hepatocellular carcinoma. Int J Clin Oncol 2007;12:363-8.

24. Wei X, Jiang Y, Zhang X, Feng S, Zhou B, et al. Neoadjuvant three-dimensional conformal radiotherapy for resectable hepatocellular carcinoma with portal vein tumor thrombus: a randomized, open-label, multicenter controlled study. J Clin Oncol 2019;37:2141-51.

25. Kim J, Lee I, Han K, Kim J, Kim K, et al. Clinical features of hepatocellular carcinoma patients undergoing resection after concurrent chemoradiation therapy. Int J Radiat Oncol Biol Phys 2012;84:S336-7.

26. Katz AW, Chawla S, Qu Z, Kashyap R, Milano MT, et al. Stereotactic hypofractionated radiation therapy as a bridge to transplantation for hepatocellular carcinoma: clinical outcome and pathologic correlation. Int J Radiat Oncol Biol Phys 2012;83:895-900.

27. O'Connor JK, Trotter J, Davis GL, Dempster J, Klintmalm GB, et al. Long-term outcomes of stereotactic body radiation therapy in the treatment of hepatocellular cancer as a bridge to transplantation. Liver Transpl 2012;18:949-54.

28. Sapisochin G, Barry A, Doherty M, Fischer S, Goldaracena N, et al. Stereotactic body radiotherapy vs. TACE or RFA as a bridge to transplant in patients with hepatocellular carcinoma. An intention-to-treat analysis. J Hepatol 2017;67:92-9.

29. Wang WH, Wang Z, Wu JX, Zhang T, Rong WQ, et al. Survival benefit with IMRT following narrow-margin hepatectomy in patients with hepatocellular carcinoma close to major vessels. Liver Int 2015;35:2603-10.

30. Rodríguez-Perálvarez M, Luong TV, Andreana L, Meyer T, Dhillon AP, et al. A systematic review of microvascular invasion in hepatocellular carcinoma: diagnostic and prognostic variability. Ann Surg Oncol 2013;20:325-39.

31. Wang L, Wang W, Yao X, Rong W, Wu F, et al. Postoperative adjuvant radiotherapy is associated with improved survival in hepatocellular carcinoma with microvascular invasion. Oncotarget 2017;8:79971-81. 